\title{
A!
}

This is an electronic reprint of the original article.

This reprint may differ from the original in pagination and typographic detail.

Chen, Kexun; Setala, Olli E.; Radfar, Behrad; Kroth, Udo; Vahanissi, Ville; Savin, Hele

\section{Harnessing Carrier Multiplication in Silicon Solar Cells Using UV Photons}

Published in:

IEEE Photonics Technology Letters

DOI:

10.1109/LPT.2021.3124307

Published: 15/12/2021

Document Version

Peer reviewed version

Please cite the original version:

Chen, K., Setala, O. E., Radfar, B., Kroth, U., Vahanissi, V., \& Savin, H. (2021). Harnessing Carrier Multiplication in Silicon Solar Cells U'sing UV Photons. IEEE Photonics Technology Letters, 33(24), 1415-1418.

https://doi.org/10.1109/LPT.2021.3124307

This material is protected by copyright and other intellectual property rights, and duplication or sale of all or part of any of the repository collections is not permitted, except that material may be duplicated by you for your research use or educational purposes in electronic or print form. You must obtain permission for any other use. Electronic or print copies may not be offered, whether for sale or otherwise to anyone who is not an authorised user. 


\title{
Harnessing Carrier Multiplication in Silicon Solar Cells Using UV Photons
}

\author{
Kexun Chen, Olli E. Setälä, Behrad Radfar, Udo Kroth, Ville Vähänissi, and Hele Savin
}

\begin{abstract}
Silicon solar cells are known to suffer from poor emitter performance that is seen as reduced external quantum efficiency at wavelengths below $500 \mathrm{~nm}$. This is due to common tradeoff between electrical and optical performance. Here we demonstrate that no such tradeoff is needed when optimized boron implantation parameters are combined with non-reflective nanostructures and atomic layer deposited $\mathrm{Al}_{2} \mathrm{O}_{3}$ surface passivation. As a result, in our solar cells the external quantum efficiency actually increases with decreasing wavelength and reaches even above $100 \%$ at short wavelengths. This result indicates that carrier multiplication caused by absorption of high energy photons could be utilized for energy production in solar cells.
\end{abstract}

Index Terms - Carrier multiplication, implantation, silicon, solar cells, reflectance, recombination, UV photons

\section{INTRODUCTION}

$\mathrm{P}$ HOTOVOLTAIC effect is the core phenomenon utilized to convert sunlight into electricity. To make the energy conversion process as effective as possible, it is important to have precise control over optical and electrical properties both at the surface and inside the photovoltaic material. This has led to multiple advances in related material research, such as reduced reflectance at the surface via utilization of nanostructures ${ }^{[1]}$, improved surface passivation using atomic layer deposited (ALD) thin films ${ }^{[2]}$ and low recombination charge collecting pn-junction (emitter) formation by ion implantation $^{[3]}$. However, despite these promising advancements, the photocurrents of silicon solar cells obtained at wavelengths below $500 \mathrm{~nm}$ (i.e. from photons absorbed within the front junction) are still far from ideal ${ }^{[4],[5]}$.

The reason for the poor performance can be sought from the interplay between the junction formation and the surface morphology. While from the optical perspective the optimal morphology would consist of nanoscale structures, it is challenging to form a high-quality emitter with such surface. This is because the surface area exposed to doping is highly enlarged compared to planar. For example, in case of diffusion, which is the most common method for emitter formation, the density of dopant atoms will easily become too high resulting in increased Auger and Shockley-Read-Hall (SRH) recombination. Therefore, a tradeoff is needed resulting in a compromise between electrical and optical performance. For instance, in today's PV industry, it is common to prioritize the emitter properties over the reflectance ${ }^{[5]}$.

One recently proposed solution to avoid the above compromise is to use so-called induced junction technology, where high doping density in the nanostructures is avoided by

This work was supported by Academy of Finland (\#331313) and by EMPIR program through the project 19ENG05 NanoWires co-financed by the Participating States and from the European Union's Horizon 2020 program. The provision of facilities by Micronova Nanofabrication Centre in Espoo, Finland is acknowledged. The work is related to the Flagship on Photonics Research and Innovation "PREIN" funded by Academy of Finland. inverting the silicon surface using highly charged dielectric layer instead of external dopant atoms ${ }^{[6]}$. Actually, this solution has resulted even in successful harnessing of carrier multiplication leading to above unity quantum efficiencies in photodetectors $^{[7]}$. Such performance would be highly attractive in solar cells as well. However, induced junction cannot be directly used in silicon solar cells due to resistive losses as the inversion layer typically has rather high sheet resistance. Another more viable method for emitter formation in solar cells is ion implantation technology, where the dopant dose is predetermined by the implantation parameters. This means that the total amount of dopants is independent of the surface area. Consequently, the presence of nanostructures will dilute the dopant density allowing accurate tuning of the electrical properties of the emitter. Indeed, there are already some promising results achieved related to this approach ${ }^{[8]}$ and it would be interesting to see if the optimized ion implantation parameters allow harnessing the full potential of the nanostructures in silicon solar cells.

In this paper we combine non-reflective nanostructures, ALD $\mathrm{Al}_{2} \mathrm{O}_{3}$ surface passivation and implanted boron emitter to fabricate n-type front junction silicon solar cells with phosphorus back-surface-field (BSF). Our focus is on the optical and electrical performance of the emitter. In addition to determining the emitter saturation current $\left(\mathrm{J}_{0 \mathrm{e}}\right)$, emitter sheet resistance $\left(R_{s h}\right)$ and contact resistance $\left(R_{c}\right)$, external quantum efficiency (EQE) is measured focusing on short wavelength region. The obtained results are compared with the most advanced front junction solar cells found from literature followed by discussion on the possible physical mechanisms behind the surprisingly high photocurrent obtained.

\section{EXPERIMENTAL}

The substrates used in this work were phosphorus doped 4inch float-zone (FZ) (100) oriented Si wafers with a resistivity of $3 \Omega \cdot \mathrm{cm}$. On one wafer, 7 implanted nanotextured front junction solar cells with phosphorus BSF with an active area of $2 \mathrm{~cm} \times 2 \mathrm{~cm}$ were fabricated. The cross section of a single solar cell is shown in Fig. 1.

The first main processing step was the nanostructure formation on the front side of the cells, which was achieved using reactive ion etching (RIE) with $\mathrm{SF}_{6} / \mathrm{O}_{2}$ plasma for 7 min at a pressure of $10 \mathrm{mTorr}$ and at a temperature of $-120^{\circ} \mathrm{C}$ with Oxford Instruments Plasmalab System 100 - ICP 180. The resulting surface morphology is shown in Fig. 1. as an inset. The height of the nanostructures is roughly 400-1000 nm, the width varies between $200-400 \mathrm{~nm}$ and the density is $\sim 20-25$

K.C, O.S, B.R, V.V, H.S are with the Aalto University, Dept. of Electronics and Nanoeng., 02150 Espoo, Finland (e-mail: firstname.surname@aalto.fi).

U.K. is with Physikalisch-Technische Bundesanstalt, AG UV- and VUVRadiometry, D-10587 Berlin, Germany (e-mail: udo.kroth@ptb.de). 
nanostructures per $\mu \mathrm{m}^{2}$. Such surface nanotexture is known to be very efficient for light absorption due to the so-called moth's eye principle ${ }^{[9]}$.

The surface texturization was followed by the formation of the emitter, the core of the device. This was realized with boron $(\mathrm{p}+)$ implantation. The implantation and the drive-in parameters were selected based on our earlier publication ${ }^{[8]}$. The implantation energy was $10 \mathrm{keV}$ and the dose was $3 \times 10^{15} \mathrm{~cm}^{-2}$. The drive-in consisted of a 20 min post-anneal in $\mathrm{N}_{2}$ ambient at a temperature of $1050^{\circ} \mathrm{C}$, followed by a 20 min dry oxidation resulting in a $13 \mathrm{~nm} \mathrm{SiO}{ }_{2}$ layer. The drive-in anneal simultaneously recovered the possible implantation damage as well as stabilized the bulk defects that can be present in the FZ wafers ${ }^{[10]}$. These emitter formation parameters have earlier been shown to result in low recombination ${ }^{[8]}$. At the backside of the wafers, phosphorus $\left(\mathrm{n}^{+}\right)$implantation was applied with the energy of $20 \mathrm{keV}$ and the dose of $4.75 \times 10^{15} \mathrm{~cm}^{-2}$. The drive-in was done together with the front side emitter. The $\mathrm{n}^{+}$layer was used for the formation of a so-called BSF to improve the backside passivation and to also allow ohmic bulk contact formation.

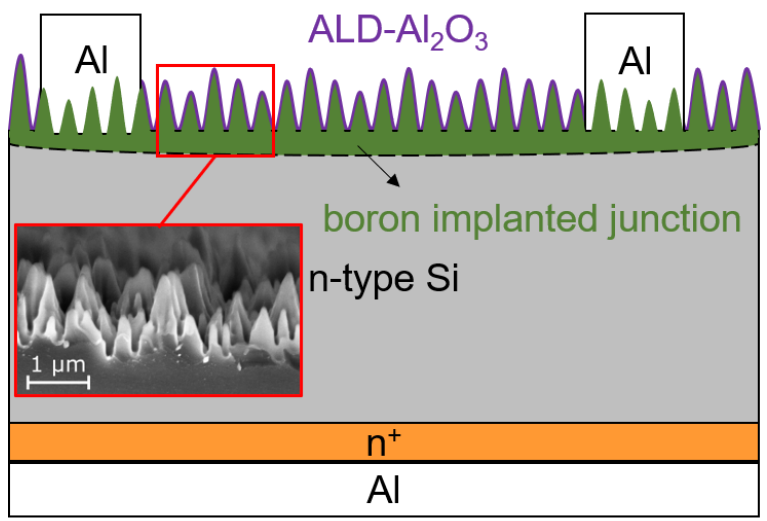

Fig. 1. Cross section of the implanted nanostructured front junction solar cells prepared in this work.

After emitter formation, it was critical to eliminate the recombination losses at the emitter surface. This was realized by removing the thin $\mathrm{SiO}_{2}$ layer resulting from drive-in oxidation and then depositing $20 \mathrm{~nm}$ thick ALD $\mathrm{Al}_{2} \mathrm{O}_{3}$ layer with Beneq TFS-500 ALD reactor at a growth temperature of $200{ }^{\circ} \mathrm{C}$ with trimethylaluminum (TMA) and water as precursors. We have shown earlier that these parameters result in high quality interface with silicon and negative $\mathrm{Al}_{2} \mathrm{O}_{3}$ charge combined with conformal coverage of the nanostructures providing excellent surface passivation ${ }^{[2]}$. The $\mathrm{Al}_{2} \mathrm{O}_{3}$ layer was then patterned using standard photolithography to make the contact openings for the local front metal contacts. Subsequently, $5 \mu \mathrm{m}$ and $1 \mu \mathrm{m}$ thick Al layers were deposited by sputtering on the front and rear sides of the wafers, respectively. This was followed again with photolithography to pattern the busbars and fingers on the front side of the cells (finger width $10 \mu \mathrm{m}$ and spacing $1000 \mu \mathrm{m}$ ). Finally, the wafers were annealed in forming gas ambient at $425^{\circ} \mathrm{C}$ for $20 \mathrm{~min}$ to sinter the $\mathrm{Al}$ contacts as well as to activate the $\mathrm{Al}_{2} \mathrm{O}_{3}$ surface passivation. The latter is needed for the improvement of interface defect density at the $\mathrm{Al}_{2} \mathrm{O}_{3} / \mathrm{SiO}_{x} / \mathrm{Si}$ interface as well as for the increase in interface charge, which improves also the field effect passivation ${ }^{[11]}$.
The characterization of the finished devices was done as follows. The EQE was measured by PTB (PhysikalischTechnische Bundesanstalt) with traceability to a radiometric standard. The reflectance (R) was obtained with integrating sphere-based surface reflectance measurements (UV-Vis-NIR Agilent Cary 5000). The internal quantum efficiency (IQE) was calculated from the EQE and reflectance values. The sheet resistance of the emitter and the contact resistance at the front were measured using CMT-SR2000N four-point-probe and separate test structures. Finally, the emitter saturation current, $\mathrm{J}_{0 \mathrm{e}}$, was extracted from quasi-steady state photoconductance measurements at an injection level of $1.5 \times 10^{16} \mathrm{~cm}^{-3}$ using Sinton WCT-120 lifetime tester.

\section{RESULTS AND DISCUSSION}

Fig. 2(a) shows the EQE of our implanted nanotextured front junction solar cells (red curve). The EQE is measured at a wavelength range of $200-400 \mathrm{~nm}$ as in this region the photons are absorbed within the first $\sim 100 \mathrm{~nm}$ below the surface, i.e., inside the emitter. This allows us to focus on the phenomena taking place in the vicinity of the front surface and within the charge collecting junction.
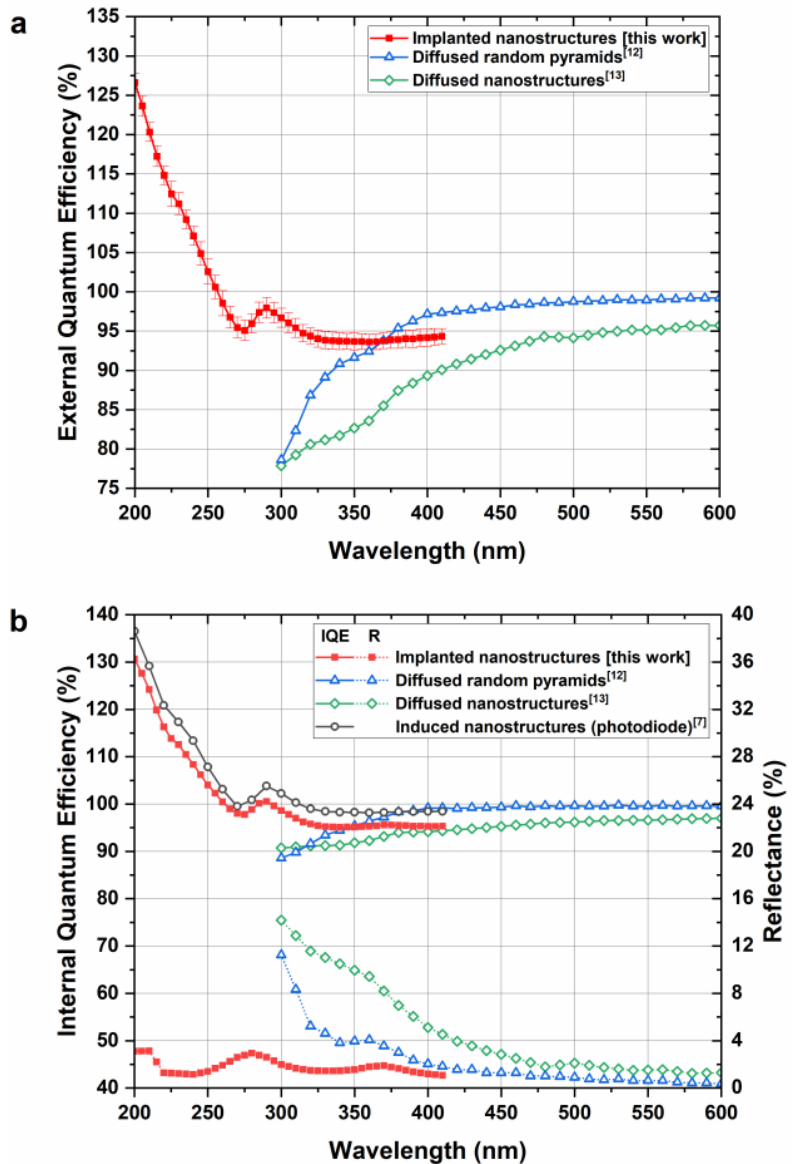

Fig. 2. (a) EQE and (b) IQE/R of the implanted nanostructured front junction solar cell prepared in this work (red). The measurement uncertainty for EQE is $\sim 1.2 \%$. Corresponding data is also shown for two record-high efficiency front junction solar cells as a reference. Additionally, IQE for induced junction photodiode is also included (black).

It is apparent from the EQE that our emitter has superior performance. We are able to convert at minimum $93.6 \%$ of the 
incoming photons to photocurrent. Furthermore, at wavelengths below $250 \mathrm{~nm}$ we can successfully collect more than one electron per incident photon. Such an interesting result could be explained by carrier multiplication, i.e., one high-energy photon is able to produce multiple electron-hole pairs. To our knowledge carrier multiplication and above unity EQE has not been observed before in solar cells.

As reference, Fig. 2(a) shows also EQE measured from solar cells that have record-high conversion efficiencies (blue ${ }^{[12]}$ and green ${ }^{[13]}$ curves). The blue curve represents data taken from a $25.8 \%$ efficient solar cell, which is the highest efficiency reported so far for a front junction solar cell ${ }^{[12]}$. This cell had random pyramid micro-structured surfaces, which were coated with $\mathrm{Al}_{2} \mathrm{O}_{3} / \mathrm{SiN}_{\mathrm{x}} / \mathrm{MgF}_{2}$ surface passivation and anti-reflection (AR) layer, and the junction was fabricated using boron diffusion. The green curve represents the highest EQE we were able to find from literature for front junction nanotextured solar cells ${ }^{[13]}$. That cell had RIE surface nanotexture, which was passivated with $\mathrm{Al}_{2} \mathrm{O}_{3} / \mathrm{SiN}_{\mathrm{x}}$ stack, and the junction was fabricated likewise by boron diffusion. When comparing our result to these curves, we see clear differences both in the shape and in the magnitude. In both reference solar cells, the UV response reduces systematically the smaller the wavelength is as opposed to the behavior of our solar cell.

In order to better understand if the difference to the reference samples in EQE results from junction recombination, surface recombination or optical properties, we investigate the IQE and $\mathrm{R}$ in the corresponding samples. The former is a measure of the electrical properties while the latter reflects the optical properties. Fig. 2(b) shows that there is indeed a clear difference in the optical properties between the samples, i.e., the reflectance in our solar cell is below $4 \%$ in the whole studied range, while both reference solar cells suffer from relatively high reflectance losses. Thus, it seems that in the both reference cases, the tradeoff in optical properties was done to secure a decent emitter performance. The high reflectance measured in the nanotextured solar cell reference may be rather surprising, but it is likely due to the challenges related to texturization of a multicrystalline wafer. Indeed, there was even an additional $\mathrm{SiN}_{\mathrm{x}}$ AR layer present on top of the nanostructures.

In addition to the optical properties, it is interesting to compare the IQE of the samples to see the possible differences related to the junction and surface recombination. As expected, based on the EQE, in the implanted sample also the IQE reaches above $100 \%$ demonstrating a junction with minimal recombination. This means that the $\mathrm{ALD} \mathrm{Al}_{2} \mathrm{O}_{3}$ is able to provide highly efficient surface passivation for nanostructured and implanted silicon surface, which most likely results from a combination of dangling bond passivation as well as field-effect passivation. Additionally, the chosen drive-in anneal is able to fully activate the dopant atoms, spread them homogeneously inside silicon nanostructures and recover the implantationinduced crystal damage. Fig. 2(b) reveals that the two reference solar cells made with diffused emitters have clear difference to the implanted emitter as they suffer from recombination losses as the IQE goes below $90 \%$ in UV. This is despite the fact that there were already some tradeoffs made regarding the optics. In any case, the recombination is likely due to non-passivated dangling bonds at the surface of the wafer combined with the recombination centers originating from non-activated dopant species in addition to possible Auger recombination. It would be interesting to see the behavior of the reference solar cells at deep UV. Nevertheless, while the recombination losses play some role in the reference samples, these results confirm that the optical properties seem to explain a majority of the differences seen in EQE between the samples. Consequently, there is no sign of carrier multiplication visible in these samples.

Fig. 2(b) includes also a third reference data (black curve) for IQE that is taken from a high-efficiency photodiode consisting of so-called induced junction ${ }^{[7]}$. This means that instead of external dopant atoms, the pn-junction is realized by inverting the surface using a highly charged dielectric layer. Because of the absence of external dopant atoms combined with efficient surface passivation, from the recombination perspective, this sample can be considered a nearly ideal junction. Therefore, it is interesting to see that the IQE curve of the implanted junction (this work) truly follows so closely that of the induced junction, including the small details seen in the curve shapes. This hints that in addition to the induced junction photodiode, the carrier multiplication is visible also in our implanted solar cell. For instance, the small bump in the curve near $290 \mathrm{~nm}$ has been suggested to be associated with generation of hot holes that are responsible for the carrier generation by impact ionization ${ }^{[14]}$ resulting from high energy photon absorption. The carrier multiplication is likely present in all silicon samples, but it can be harnessed only if the recombination losses are minimized.

In order to obtain a high conversion efficiency in a solar cell, it is not enough to have high photocurrent. Other important parameters include junction $R_{s h}$ as well as $R_{c}$ between the junction and the metal layer as they both contribute to the resistive losses. They are also closely connected to each other, as in general, the lower the $\mathrm{R}_{\mathrm{sh}}$ the lower the $\mathrm{R}_{\mathrm{c}}$. For industrially relevant front junction solar cells $R_{\text {sh }}$ around $120-170 \Omega /$ sq or lower is preferred, otherwise ohmic contact formation becomes impossible with screen printing and current transport and resistive losses at the emitter become too high ${ }^{[15]}$. An alternative is to make selectively doped local areas with high dopant density, but this requires much more complicated and expensive fabrication ${ }^{[12]}$. The sheet resistance of our solar cell is $110 \Omega / \mathrm{sq}$, which already meets the current requirement from the industry. Consequently, the contact resistance measured in our cell was as low as $0.1 \mathrm{~m} \Omega \cdot \mathrm{cm}^{2}$, which is low enough to not limit the cell efficiency.

As mentioned earlier, the low sheet resistance is preferred for ohmic contact formation, but it often comes with a tradeoff with increased emitter recombination due to a higher amount of dopant atoms and related Auger and SRH recombination. We demonstrate this in Fig. 3, which presents so-called emitter equilibrium recombination current density ${ }^{[16]}, \mathrm{J}_{0 e}$, as a function of emitter sheet resistance in the same samples than presented in Fig. 2. $\mathrm{J}_{0 \mathrm{e}}$ is a widely used recombination parameter in photovoltaics that takes into account all recombination processes taking place inside the junction as well as at the surface. Thus, it provides supporting data for the IQE presented in Fig. 2(b). For high-efficiency solar cells, $\mathrm{J}_{0 \mathrm{e}}$ should be well below $50 \mathrm{fA} / \mathrm{cm}^{2}{ }^{[13]}$. Indeed, we can see that all the cells considered in this study fulfil this condition. Additionally, we see a clear correlation between the sheet resistance and the recombination activity. For instance, although an outstanding 
$\mathrm{J}_{0 \mathrm{e}}$ of $7.5 \mathrm{fA} / \mathrm{cm}^{2}$ and $3 \mathrm{fA} / \mathrm{cm}^{2}$ can be reached in the diffused random pyramid textured and the induced junction samples, they both come with a tradeoff in $\mathrm{R}_{\mathrm{sh}}(300 \Omega / \mathrm{sq}$ and $8000 \Omega / \mathrm{sq}$, respectively). Similarly, while the nanotextured $\mathrm{mc}-\mathrm{Si}$ reference (green curve) had a low $\mathrm{R}_{\mathrm{sh}}(90 \Omega / \mathrm{sq})$, $\mathrm{J}_{0 \mathrm{e}}$ was increased to $50 \mathrm{fA} / \mathrm{cm}^{2}$. Finally, our solar cell shows impressive $\mathrm{J}_{0 \mathrm{e}}$ of $33 \mathrm{fA} / \mathrm{cm}^{2}$ considering the $\mathrm{R}_{\mathrm{sh}}$ of $110 \Omega / \mathrm{sq}$. We have also included ion implanted planar surface in Fig. 3. as an additional reference sample (open red square). It is interesting to note that with identical implantation parameters (including fixed implantation dose) $\mathrm{J}_{0 \mathrm{e}}$ is lower in nanostructured silicon than in the planar counterpart ${ }^{[8]}$ - this confirms our hypothesis that the dopant atoms are diluted in the nanostructures when using implantation. Consequently, the control of the dopant density by implantation is much easier than via diffusion.

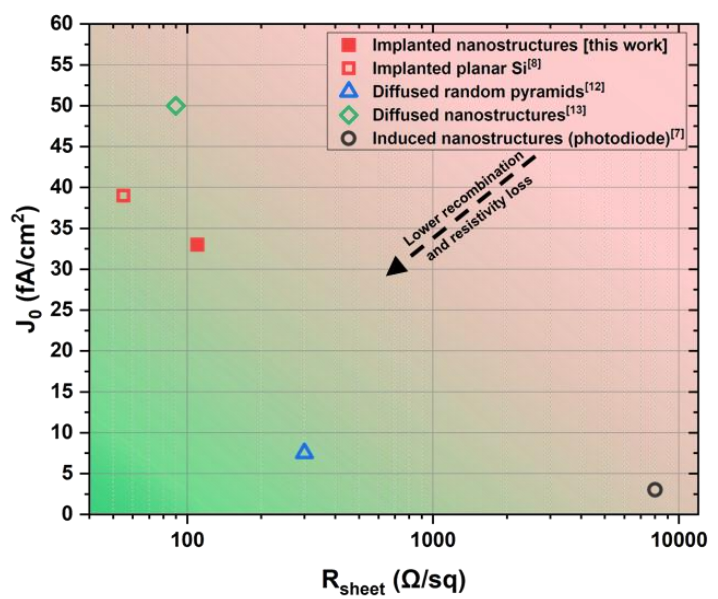

Fig. 3. Emitter saturation current $\left(\mathrm{J}_{0 \mathrm{e}}\right)$ of the implanted nanostructured and planar sample as well as the two reference solar cell emitters and an induced junction as a function of $R_{\text {sheet }}$.

This work presented record high EQE in the front side junction, which is an important prerequisite for high efficiency solar cells. The next logical step is to integrate similar front junction to the solar cells that have shown record-high performance at the rear side such as Tunnel Oxide Passivated contact (Topcon) ${ }^{[3]}$ or Passivated Emitter and Rear Cell (PERC) ${ }^{[4]}$ structures instead of using a basic phosphorus BSF structure used in this work. We see no reason why such combination would not be resulting in record high energy conversion efficiency as a recent study shows that the reflectance losses, Auger recombination, front surface recombination and junction-related resistive losses contribute to several percentage absolute efficiency drop in total in the record-high front junction solar cells ${ }^{[12]}$. Of course, the very high UV response below $300 \mathrm{~nm}$ may not be utilizable in terrestrial PV applications as AM1.5 spectrum starts at $300 \mathrm{~nm}$ and the portion of the UV radiation is relatively small in the sun spectrum arriving to earth. Furthermore, the cut-off wavelength of typical cover glass and EVA in a current solar panel is around $300 \mathrm{~nm}$. However, in space applications in AM0 there is a lot of UV radiation and the sun spectrum extends even below 250 nm. Another technology that could benefit from high UV response is the up-conversion solar cells, ${ }^{[17]}$ where the incoming lower energy photons are converted to UV photons for which the response would be higher than for, e.g., photons in the visible wavelength range.

\section{CONCLUSION}

In conclusion, we have demonstrated experimentally that carrier multiplication caused by absorption of UV photons can be utilized in crystalline silicon solar cells, which resulted in external quantum efficiency close to and even above unity. This was possible with optimized ion implantation parameters that allowed harnessing the full potential of the non-reflecting nanostructures without compromising the electrical performance of the emitter. In terrestrial PV this result should enable increasing the record efficiencies by several tenths of percent absolute while in space applications the increase in efficiency could be even higher ${ }^{[12]}$.

\section{REFERENCES}

[1] A. A. Elsayed, Y. M. Sabry, F. Marty, T. Bourouina, and D. Khalil, "Optical modeling of black silicon using an effective medium/multi-layer approach", Opt. Express, vol. 26, no. 10, pp. 13443-13460, May 2018.

[2] P. Repo, A. Haarahiltunen, L. Sainiemi, M. Yli-Koski, H. Talvitie, M. C. Schubert and H. Savin, "Effective Passivation of Black Silicon Surfaces by Atomic Layer Deposition”, IEEE J. Photovolt., vol. 3, no. 1, pp. 90-94, Jan. 2013.

[3] A. D. Upadhyaya, et al., "Ion-Implanted Screen-Printed n-Type Solar Cell With Tunnel Oxide Passivated Back Contact", IEEE J. Photovolt., vol. 6, no. 1, pp. 153-158, Jan. 2016.

[4] J Y. Lv, Y. F. Zhuang, W. J. Wang, W. W. Wei, J. Sheng, S. Zhang and W. Z. Shen, "Towards high-efficiency industrial p-type mono-like Si PERC solar cells", Sol. Energy Mater. Sol. Cells, vol. 204, pp. 110202, Jan. 2020.

[5] D. Yan, S. P. Phang, Y. Wan, C. Samundsett, D. Macdonald and A. Cuevas, "High efficiency n-type silicon solar cells with passivating contacts based on PECVD silicon films doped by phosphorus diffusion", Sol. Energy Mater. Sol. Cells, vol. 193, pp. 80-84, May 2019.

[6] M. A. juntunen, J. Heinonen, V. Vähänissi, P. Repo, D. Valluru, and H. Savin, "Near-unity quantum efficiency of broadband black silicon photodiodes with an induced junction", Nat. Photonics, vol. 10, pp. 777781, Nov. 2016.

[7] M. Garin, J. Heinonen, L. Werner, T. P. Pasanen, V. Vähänissi, A. Haarahiltunen, M. A. Juntunen and H. Savin, "Black-Silicon Ultraviolet Photodiodes Achieve External Quantum Efficiency above 130\%", Phys. Rev. Lett., vol. 125, no. 11, pp. 117702, Sep. 2020.

[8] G. von Gastrow, P. Ortega, R. Alcubilla, S. Husein, T. Nietzold, M. Bertoni, and H. Savin "Recombination processes in passivated boron-implanted black silicon emitters", J. Appl. Phys., vol. 121, pp. 185706, May 2017.

[9] P. B. Clapham, and M. C. Hutley, "Reduction of Lens Reflexion by the "Moth Eye" Principle", vol. 244, pp. 281-282, Aug. 1973.

[10] N. E. Grant, V. P. Markevich, J. Mullins, A. R. Peaker, F. Rougieux, "Permanent annihilation of thermally activated defects which limit the lifetime of float-zone silicon", Phys. Status Solidi A, vol. 213, no. 11, pp. 2844-2849, Nov. 2016.

[11] G. Dingemans, M. C. M. van de Sanden and W. M. M. Kessels, "Influence of the Deposition Temperature on the c-Si Surface Passivation by A12O3 Films Synthesized by ALD and PECVD", Electrochem. Solid-State Lett., vol. 13, no. 3, pp. H76-H79, Jan. 2010.

[12] A. Richter, R. Müller, J. Benick, F. Feldmann, B. Steinhauser, C. Reichel, "Design rules for high-efficiency both-sides-contacted silicon solar cells with balanced charge carrier transport and recombination losses", Nat. Energy, vol. 6, pp. 429-438, Apr. 2021.

[13] J. Benick et al., "High-Efficiency n-Type HP mc Silicon Solar Cells", IEEE J. Photovolt., vol. 7, no. 5, pp. 1171-1175, Sept. 2017.

[14] S. Kolodinski, J. H. Werner, T. Wittchen, and H. J. Queisser, "Quantum efficiencies exceeding unity due to impact ionization in silicon solar cells", Appl. Phys. Lett., vol. 63, pp. 2405, Aug. 1993.

[15] E. Lohmüllern, S. Werner, R. Hoenig, J. Greulich and F. Clement, "Impact of boron doping profiles on the specific contact resistance of screen printed Ag-Al contacts on silicon”, Sol. Energy Mater. Sol. Cells, vol. 142, pp. 211, Nov. 2015

[16] A. Cuevas, "The Recombination Parameter J J", Energy Procedia, vol. 55, pp. 53-62, Sept. 2014.

[17] T. Trupke and M. A. Green, "Improving solar cell efficiencies by downconversion of high-energy photons", J. Appl. Phys., vol. 92, pp. 1668, May 2002. 